\title{
PERFORMANCE ANALYSIS FOR A SUBSPACE DECOMPOSITION POINT-SOURCE IMAGE RESTORATION ALGORITHM
}

\author{
Brian D. Jeffs and Metin Gunsay, members IEEE
}

\author{
Brigham Young University, Department of Electrical and Computer Engineering \\ 459 Clyde Building, Provo Utah 84602
}

\begin{abstract}
This paper addresses the problem of resolving and localizing blurred point sources in intensity images, such as atmospherically blurred star images. It is shown that this problem can be modeled in a form similar to that used for sensor array data in direction of arrival (DOA) estimation. A new superresolving restoration algorithm is described as a generalization of eigenstructure decomposition methods popular in DOA estimation. The algorithm includes a signal subspace rank enhancement technique that works in the presence of non-uniform element weighting due to blur. A theoretical analysis of source localization error is presented, and an expression predicting error performance is derived. Restoration examples are presented illustrating the accuracy of the error analysis and demonstrating algorithm performance.
\end{abstract}

\section{Introduction}

Point source image restoration involves resolving individual points, or impulses, in an image that has been corrupted by convolution with some blurring function. For this special case of image restoration, it is known or assumed a priori that the true image is sparse or point like. The point spread function (PSF) of the imaging system can merge closely located point sources to the extent that they can not be resolved in the presence of noise without some form of superresolution provided by the restoration algorithm.

Point image restoration is applicable in the fields of astronomical image restoration, biomedical imaging and echo resolution. Deblurring of star fields is one of the major applications. Blur in long exposure astronomical star images may be due to atmospheric turbulence, misfocus, poor telescope tracking, finite aperture size, or other optical distortion effects. Atmospheric turbulence can cause nearby stars to become blurred beyond resolution so as to appear as a single star.

The eigenvector based approach discussed here depends on the underlying point-like structure of the true image, and is closely related to methods used for source localization in sensor array processing. By transforming the problem into the frequency domain, we exploit the fact that spatial shifts of a finite support blurring function result in phase shifts across the elements of the frequency domain image, and yield data structurally similar to sensor array data seen in DOA estimation.

This work supported in part by NSF grant MI9110187.
This algorithm was presented by the authors at ICASSP-94 [1], and will be reviewed here. The emphasis of this paper is a new performance analysis which permits prediction of restoration error as a function of algorithm parameters.

The following observation model for an image (or image sequence) observed at time $t$. will be used in this development

$$
g(t)=D f+\eta(t)
$$

where $g$ is the row-scanned observation vector, $D$ is a doubly block circulant 2-D convolution matrix whose columns are formed from spatially shifted copies of the row scanned blurring function, $f$ is the uncorrupted deterministic image vector, and $\eta$ is the additive observation noise vector. The doubly block circulant structure of $\boldsymbol{D}$ implies that source position shifts are mapped to 2-D phase shifts in the frequency domain. The circular convolution assumed by this model introduces no error as compared to the true linear convolution observed in the image data, if the point sources of interest are no closer to image edges than the width of the PSF region of support.

Note that in equation (1), $f$ is independent of time. This model is analogous to the fully coherent scene case found in the DOA literature, and requires special techniques to build up the degenerate rank of the associated autocovariance matrix before eigendecomposition methods may be applied. We introduce a new 2-D rank enhancement method which is an extension to 1-D array smoothing as proposed by T. Shan et al [2].

\section{Algorithm Theoretical Development}

In this section we transform the image restoration problem of equation (1) into a form which can be solved with traditional eigenstructure based DOA estimation techniques.

\subsection{Frequency Domain Signal Model}

Since $f$ is sparse, we may replace $D f$ with $A u$, where $u$ contains the intensity coefficients of all the non-zero elements of $f$, and $A$ contains only those columns of $D$ which correspond to elements of $\boldsymbol{u}: \boldsymbol{u}$ has length $P$, equal to the number of point sources in the image.

$$
\boldsymbol{g}(t)=\boldsymbol{A u}+\eta(t) .
$$

In order to map spatial shifts in source position to phase shifts, (2) is transformed into the frequency domain [1][3]

$$
\tilde{\mathbf{g}}(t)=\tilde{A} \boldsymbol{u}+\tilde{\eta}(t)
$$


where $\sim$ indicates the 2-D DFT is computed prior to row scanning the images, and each of the columns of the matrix A. Columns of $\tilde{A}$ have identical magnitude, but a different progressive phase because columns of $\boldsymbol{A}$ are related by block circulant shifting of the PSF. We can express $\tilde{A}$ as the product of a diagonal matrix $\boldsymbol{H}$, and a matrix $\boldsymbol{V}$ which contains the phase (position) information for each of the columns of $\tilde{\boldsymbol{A}}$

$$
\tilde{\boldsymbol{A}}=\boldsymbol{H}\left[\boldsymbol{v}_{x_{1}, y_{1}}\left|v_{x_{2}, y_{2}}\right| \cdots \mid v_{x_{p}, y_{P}}\right]=\boldsymbol{H} \boldsymbol{V}
$$

$\boldsymbol{H}$ is the frequency domain representation of the unshifted PSF, i.e. $\boldsymbol{H}=\operatorname{diag}\{\operatorname{FFT}(\boldsymbol{d})\}$ where $\boldsymbol{d}$ is the first column of $\boldsymbol{D}$. Each frequency domain position vector, $v_{x_{P}, y_{P}}$, corresponds to an image with a single unblurred point at location $\left(x_{p}, y_{p}\right)$,

$$
\begin{aligned}
v_{x, y}= & {\left[e^{-j 2 \pi\left(\frac{0 x}{M_{1}}+\frac{0 y}{M_{2}}\right)}, e^{-j 2 \pi\left(\frac{0 x}{M_{1}}+\frac{1 y}{M_{2}}\right)}, \cdots, e^{-j 2 \pi\left(\frac{0 x}{M_{1}}+\frac{\left(M_{2}-1\right) y}{M_{2}}\right)},\right.} \\
& \left.\cdots e^{-j 2 \pi\left(\frac{\left(M_{1}-1\right) x}{M_{1}}+\frac{0 y}{M_{2}}\right)}, \cdots, e^{-j 2 \pi\left(\frac{\left(M_{1}-1\right) x}{M_{1}}+\frac{\left(M_{2}-1\right) y}{M_{2}}\right)}\right]
\end{aligned}
$$

The autocovariance matrix of $\tilde{\boldsymbol{g}}$ can now be represented as

$$
\boldsymbol{R}_{g}=\boldsymbol{H} \boldsymbol{V} \boldsymbol{R}_{\boldsymbol{u}} \boldsymbol{V}^{H} \boldsymbol{H}^{H}+\boldsymbol{I} \sigma_{\eta}^{2} .
$$

\subsection{Generalized 2-D Smoothing}

Equation (6) is similar to the covariance model used in coherent scene DOA problems [2]. The signal subspace of $\boldsymbol{R}_{\tilde{g}}$, represented by the first term in equation (6), has a rank of one because the magnitudes of the point sources are not time varying. Rank must be increased to $P$ for eigenvector based methods to work. Unfortunately, blur operator, $\boldsymbol{H}$, prohibits using conventional array smoothing, as typically applied to uniform line arrays [2]. We introduce a new rank enhancement method as a generalization of spatial smoothing. Generalized 2-D smoothing is accomplished by weighted averaging in the frequency domain over the autocovariance matrices of sub-images.

Figure 1 shows how these sub-images are extracted from a larger image and how they are arranged into a vector. The observed frequency domain sub-images are given by

$$
\begin{aligned}
\tilde{\boldsymbol{g}}_{[m, n]}(t)= & {\left[\tilde{g}_{m, n}, \tilde{g}_{m, n+1}, \cdots, \tilde{g}_{m, \Theta_{2}}, \tilde{g}_{m+1, n}, \tilde{g}_{m+1, n+1}, \cdots\right.} \\
& \left., \tilde{g}_{m, \Theta_{2}}, \cdots, \tilde{g}_{\Theta_{1}, n}, \tilde{g}_{\Theta_{1}, n+1}, \cdots, \tilde{g}_{\Theta_{1}, \Theta_{2}}\right] \\
\tilde{\boldsymbol{g}}_{[m, n]}(t)= & \boldsymbol{H}_{[m, n]} \boldsymbol{V}_{[m, n]} \boldsymbol{u}+\tilde{\eta}(t)
\end{aligned}
$$

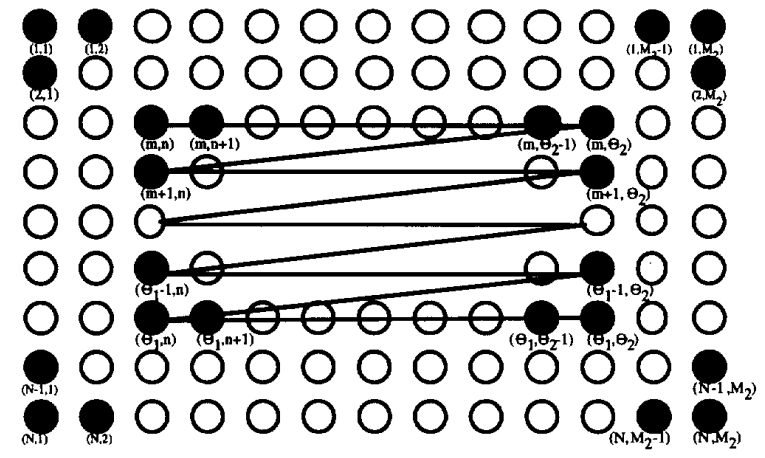

Figure 1. Row scanned sub-image at $(m, n)$.

$V$ is not Vandermonde as in the uniform line array case, but fortunately there is a simple relationship between all of the $\boldsymbol{V}_{[m, n]}$ which may be exploited for smoothing. $V_{[m, n]}=V_{[1,1]} C_{[m, n]}$, where

$$
\begin{aligned}
C_{[m, n]}= & \left\{e^{-j 2 \pi\left(\frac{x_{1}(m-1)}{M_{1}}+\frac{y_{1}(n-1)}{M_{2}}\right)}, e^{-j 2 \pi\left(\frac{x_{2}(m-1)}{M_{1}}+\frac{y_{2}(n-1)}{M_{2}}\right)},\right. \\
& \left.\cdots, e^{-j 2 \pi\left(\frac{x_{P}(m-1)}{M_{1}}+\frac{y_{P}(n-1)}{M_{2}}\right)}\right\}
\end{aligned}
$$

Assuming the noise is uncorrelated from pixel to pixel, the autocovariance matrix of $\tilde{\boldsymbol{g}}_{[m, n]}$ is

$$
\boldsymbol{R}_{[m, n]}=\boldsymbol{H}_{[m, n]} \boldsymbol{V}_{[1,1]} \boldsymbol{C}_{[m, n]} \boldsymbol{R}_{u} \boldsymbol{C}_{[m, n]}^{H} \boldsymbol{V}_{[1,1]}^{H} \boldsymbol{H}_{[m, n]}^{H}+\boldsymbol{I} \sigma_{\eta}^{2}
$$

The $\boldsymbol{H}_{[m, n]}$ terms in (8) are dependent on sub-image indices and prohibit smoothing. To overcome this difficulty and to introduce a regularization operator to control noise amplification, we define a "smoothing regularization matrix", $Q . Q$ is chosen to be an arbitrary, constant, diagonal matrix. Given a choice for $\boldsymbol{Q}$, weighting matrices, $\boldsymbol{W}_{[m, n]}$, are computed as solutions to

$$
\boldsymbol{Q}=\boldsymbol{W}_{[m, n]} \boldsymbol{H}_{[m, n]} \quad \forall[m, n] .
$$

The generalized smoothed covariance matrix is defined as

$$
\boldsymbol{R}=\frac{1}{L} \sum_{[m, n] \in B} \boldsymbol{W}_{[m, n]} \boldsymbol{R}_{[m, n]} \boldsymbol{W}_{[m, n]}^{H}
$$

where $B$ is the set of sub-images included in smoothing. When equations (8) and (9) are substituted into (10), the sub-image dependent $\boldsymbol{H}_{[m, n]}$ are replaced by the constant matrix $Q$, and the expression may be factored

$$
\begin{aligned}
\boldsymbol{R} & =\boldsymbol{Q} \boldsymbol{V}_{[1,1]} \overline{\boldsymbol{R}}_{u} \boldsymbol{V}_{[1,1]}^{H} Q^{H}+\sigma_{\eta}^{2} \overline{\boldsymbol{W}} \\
\overline{\boldsymbol{R}}_{u} & =\frac{1}{L} \sum_{[m, n] \in B} \boldsymbol{C}_{[m, n]} \boldsymbol{R}_{u}\left(\boldsymbol{C}_{[m, n]}\right)^{H}, \overline{\boldsymbol{W}}=\frac{1}{L} \sum_{[m, n] \in B}\left|\boldsymbol{W}_{[m, n]}\right|^{2}
\end{aligned}
$$


As in the traditional DOA problem, it can be shown that with the proper choice of sub-images, the rank of $\overline{\boldsymbol{R}}_{u}$ will increase to $\boldsymbol{P}$ for each additional sub-image which is included in the average [1][3]. Thus eigenvector methods can be directly applied to the smoothed autocovariance matrix $\boldsymbol{R}$.

\subsection{Restoration Algorithm}

Given $\boldsymbol{R}$, a point source image restoration algorithm based on MUSIC [4] may be developed. The smoothed sample covariance matrix is computed as an estimate of $\boldsymbol{R}$

$$
\hat{\boldsymbol{R}}=\frac{1}{L \Omega} \sum_{[m, n] \in B} \sum_{l=1}^{\Omega}\left(\boldsymbol{W}_{[m, n]} \tilde{\boldsymbol{g}}_{[m, n]}(l T)\right)\left(\boldsymbol{W}_{[m, n]} \tilde{\boldsymbol{g}}_{[m, n]}(l T)\right)^{H}
$$

where $T$ is the interval between time samples and $\Omega$ is the total number of samples. Due to the averaging implicit in smoothing, single snapshot data $(\Omega=1)$ is acceptable.

Estimates of the signal and noise subspaces, $\hat{\boldsymbol{E}}_{s}$ and $\hat{E}_{n}$, are found by solving the generalized eigenvector problem,

$$
\hat{\boldsymbol{R}}\left[\hat{\boldsymbol{E}}_{s} \mid \hat{\boldsymbol{E}}_{\eta}\right]=\overline{\boldsymbol{W}}\left[\hat{\boldsymbol{E}}_{s} \mid \hat{\boldsymbol{E}}_{\eta}\right] \hat{\Lambda}
$$

If the number of sources in the scene is known to be $P$, then $\hat{E}_{s}$ contains eigenvectors corresponding to the $P$ largest eigenvalues in $\hat{\Lambda}$. The minimum description length (MDL) method [7] was used to estimate $P$ in computing results shown in Section 4.

The restored image is computed from the modified MUSIC spectrum, defined as:

$$
\hat{f}(x, y)=\frac{\left(Q v_{x, y}\right)^{H}\left(Q v_{x, y}\right)}{\left(Q v_{x, y}\right)^{H} \hat{\boldsymbol{E}}_{\eta} \hat{\boldsymbol{E}}_{\eta}^{H}\left(Q \boldsymbol{v}_{x, y}\right)}
$$

where $v_{x, y}$ is the position vector of equation $(5) . \hat{f}(x, y)$ is a continuous function of $(x, y)$, and may be computed at any desired sample spacing. Peaks in the spectrum correspond to estimated point positions.

\subsection{Designing $Q$}

$Q$ must be specified by the user and serves several important functions. Without it, smoothing in the presence of a blurring function would not be possible. Though a number choices have been shown to work acceptably, the following $Q$ specification stabilizes the solution with respect to noise amplification in the reconstruction, and whitens the noise subspace [3]. Equation (15) was used in the results to follow.

$$
Q_{i, i}=\left\{\begin{array}{cc}
\sqrt{\frac{L}{\sum_{[m, n] \in B} \frac{1}{\left|\left(\boldsymbol{H}_{[m, n]}\right)_{i, i}\right|^{2}}}} & \text { if }\left|\left(\boldsymbol{H}_{[m, n]}\right)_{i, i}\right|>0 \\
0 & \forall[m, n] \in B \\
0 & \text { otherwise }
\end{array}\right.
$$

\section{Performance Analysis}

For point source image restoration, the most important measure of performance is usually the spatial accuracy in placement of the located points. A metric such as SNR improvement would be inappropriate, since because the image is assumed to be point-like, only peaks of $\hat{f}(x, y)$ are significant, and the image background noise level can be neglected. We will use mean squared error (MSE) in position for the $p$ th point as our performance criterion,

$$
\overline{\Delta x_{p}^{2}} \equiv E\left\{\left(\hat{x}_{p}-x_{p}\right)^{2}\right\}
$$

where $\hat{x}_{p}$ is the estimated position, and $x_{p}$ is the true value. Without loss of generality, we consider only the $x$ component of the $p$ th detected peak in $\hat{\boldsymbol{f}}\left(\hat{x}_{p}, \hat{y}_{p}\right)$.

A number of parameters can affect performance of the algorithm, including the amount of averaging used in smoothing, the number of snapshots, image size, the choice of $Q$, and the SNR. The analysis presented here seeks to quantify the effects of these variables on resolution capabilities of the algorithm by generalizing methods reported in the literature for smoothed eigenstructure based DOA estimation [3][5].

A first order approximation used by Rao and Hari and others for position error [5] can be generalized as follows to include $\boldsymbol{Q}$ and the position scan vector, $\boldsymbol{v}_{x}[3]$

$$
\overline{\Delta x_{p}^{2}} \approx \frac{\left[\begin{array}{l}
E\left\{\dot{\boldsymbol{v}}_{x}^{H} \boldsymbol{Q} \boldsymbol{E}_{\eta} \breve{\boldsymbol{E}}_{\eta}^{H} \boldsymbol{Q} \boldsymbol{v}_{x} \boldsymbol{v}_{x}^{H} \boldsymbol{Q} \breve{E}_{\eta} \boldsymbol{E}_{\eta}^{H} \boldsymbol{Q} \dot{\boldsymbol{v}}_{x}\right\} \\
+E\left\{\operatorname{Re}\left\{\dot{\boldsymbol{v}}_{x}^{H} \boldsymbol{Q} \boldsymbol{Q} \boldsymbol{E}_{\eta} \breve{\boldsymbol{E}}_{\eta}^{H} \boldsymbol{Q} \boldsymbol{v}_{x} \dot{\boldsymbol{v}}_{x}^{H} \boldsymbol{Q} \boldsymbol{E} \boldsymbol{E}_{\eta} \breve{\boldsymbol{E}}_{\eta}^{H} \boldsymbol{Q} \boldsymbol{v}_{x}\right\}\right\}
\end{array}\right]}{2\left[\dot{\boldsymbol{v}}_{x}^{H} \boldsymbol{Q} \boldsymbol{E} \boldsymbol{E}_{\eta} \boldsymbol{E}_{\eta}^{H} \boldsymbol{Q} \dot{\boldsymbol{v}}_{x}\right]^{2}}
$$

where $\dot{v}_{x}=\frac{\partial v_{x, y}}{\partial x}$, and $\breve{E}_{\eta}=\hat{E}_{\eta}-E_{\eta}$.

The difficulty with equation (17) is that the true and perturbation noise subspace terms $\left(\boldsymbol{E}_{\eta}\right.$ and $\left.\breve{\boldsymbol{E}}_{\eta}\right)$ cannot be readily computed from the observation, $\tilde{\boldsymbol{g}}_{[m, n]}$.

Beginning with the development of Rao and Hari, the following approximation for point position error was derived, which uses only observed or computed quantities. Note that unlike the approach of [5], this new expression does not require that noise be distributed i.i.d. complex circular Gaussian, and permits non-uniform element weighting. 


$$
\begin{aligned}
& \overline{\Delta x_{p}^{2}} \approx \frac{1}{2 \Omega L^{2}\left[\dot{v}_{x}^{H} Q \hat{E}_{n} \hat{E}_{n}^{H} Q \dot{v}_{x}\right]^{2}} . \\
& \sum_{\forall \mid m, n] \in B}^{L} \sum_{\forall[i, k] \in B}^{L}\left[\begin{array}{l}
\alpha_{x}^{H} N_{[m, n],[i, k]} \alpha_{x} \beta^{H} \boldsymbol{R}_{[m, n],[i, k]} \beta \\
+\operatorname{Re}\left\{\alpha^{H} N_{[m, n],[i, k]}^{\prime} \alpha^{*} \beta^{H} S_{[m, n],[i, k]}^{H} \beta\right\}
\end{array}\right]
\end{aligned}
$$

where $\alpha_{x}^{H}=\dot{\boldsymbol{v}}_{x}^{H} \boldsymbol{Q} \boldsymbol{E}_{\eta} \boldsymbol{E}_{\eta}^{H}, \beta=\boldsymbol{E}_{s} \Lambda_{s}^{-1} \boldsymbol{E}_{s}^{H} \boldsymbol{Q v}$,

$$
N_{[m, n],[i, k]}=\boldsymbol{W}_{[m, n]} \tilde{\eta}_{[m, n]} \tilde{\eta}_{[i, k]}^{H} \boldsymbol{W}_{[i, k]}^{H} \text {, }
$$$$
\boldsymbol{N}_{[m, n],[i, k]}^{\prime}=\boldsymbol{W}_{[m, n]} \tilde{\eta}_{[m, n]} \tilde{\eta}_{[i, k]}^{T} \boldsymbol{W}_{[i, k]}^{T} \text {, }
$$$$
\boldsymbol{R}_{[m, n],[i . k]}=\boldsymbol{W}_{[m, n]} \tilde{\boldsymbol{g}}_{[m, n]} \tilde{\boldsymbol{g}}_{[i, k]}^{H} \boldsymbol{W}_{[i, k]}^{H} \text {, and }
$$$$
\boldsymbol{S}_{[m, n],[i, k]}=\boldsymbol{W}_{[m, n]} \tilde{\boldsymbol{g}}_{[m, n]} \tilde{\boldsymbol{g}}_{[i, k]}^{T} \boldsymbol{W}_{[i, k]}^{T},
$$

With equation (18) it is possible to predict performance for a given set of data, perform a trade-off analysis between sub-image size and the amount of smoothing achieved, and to gain insight into the effects of design choices for $Q$ [3]. Figure 2 presents a simple two star example of predicting MSE in position as a function of the number (L) of subimages used in smoothing. $\mathrm{L}$ is determined by sub-image size. All possible sub-image shifts in the observed 12 by 12 pixel image are used, so as sub-image size decreases, $\mathrm{L}$ increases. Point sources were located at $(x, y)$ coordinates of $(5,7)$ and $(7,5)$, with a circular Gaussian blur of one pixel standard deviation. i.i.d Gaussian noise was added for an SNR of $40 \mathrm{~dB}$. Figure 2 also plots the actual error averaged over 300 trials with different noise samples (solid line), and demonstrates that equation (18) provides an excellent approximation to the true error. Figure 3 shows how equation (18) can be used to evaluate position error as a function of blur extent and the choice of $Q$. In Figure 3a, a circular Gaussian blur function with different standard deviation widths (w) was evaluated against the number of sub-images. In 3b, performance for three different $Q$ values is presented. The observed image was the same as in Figure 2 .

\section{Experimental Results}

Figures 4 and 5 present two examples of restoration for synthetic star images which are useful in evaluating performance because the underlying true image is known. The first synthetic case demonstrates the superresolution capability of the algorithm. Figure $4 \mathrm{a}$ is a cluster where two stars are separated diagonally by two pixels. The stars are blurred with a Gaussian shaped PSF, i.i.d. Gaussian noise is added for a SNR of $40 \mathrm{~dB}$, and the blurred image is sampled at $1 / 4$ the original pixel resolution (in each direction) to yield the observed image in Figure $4 \mathrm{~b}$. In this image, the star pair has a separation of only $1 / 2$ pixel. Figure $4 \mathrm{c}$ shows the resulting MUSIC spectrum, scanned at 4 times the resolution of Figure $4 \mathrm{~b}$. Note that the apparent star between the two true stars is actually a saddle point at lower intensity, and is not a peak. Figure $4 d$ shows the location of peaks in $4 \mathrm{c}$, and correctly locates all three stars.

Figure 5 presents an example performance comparison between the new algorithm and two common image restoration approaches. Figure 5a shows the observed image for a three star cluster, blurred and noise corrupted as in Figure 4. Figure $5 \mathrm{~b}$ shows a least squares reconstruction which fails miserably since this method favors smooth solutions and does not use the prior knowledge that the true image is point-like. Figure $5 \mathrm{c}$ is a restoration using the CLEAN algorithm [6] which is well suited for star images, and produces point-like results. However, since the blur is severe, this method biases the star positions, and adds a fourth star artifact. Figure 5d is the peak-picked MUSIC spectrum using the new algorithm. Computed positions exactly agree (within one pixel quantization) with the true image.

\section{Summary}

It has been shown that a duality exits between point source image restoration and the coherent DOA problem.

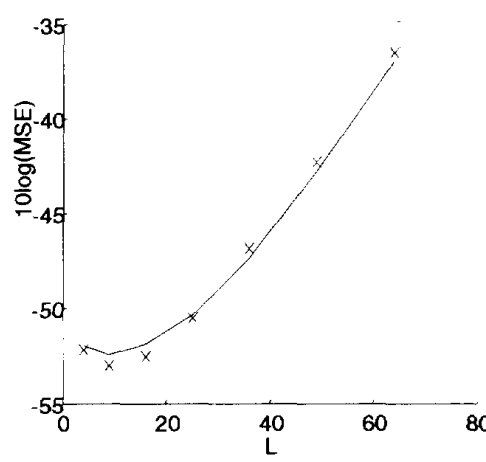

Figure 2. Mean squared position error as a function of number of sub-images. $X$ 's indicate values from eqn. (18).

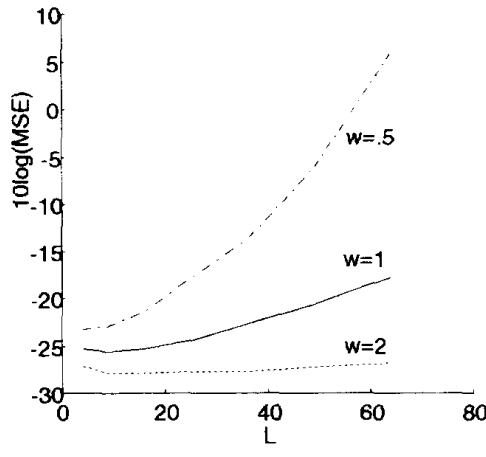

a)

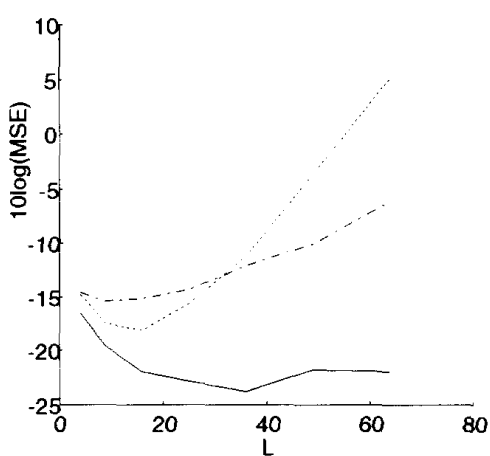

b)

Figure 3. a) Position error as a function of blur width (w). b) Position error as a function of the choice for $Q$; dash-dot line: $Q$ as per equation (15), dotted line: $Q=I$, and solid line: $Q_{i, i}=\min _{[m, n] \in B}\left|\left(H_{[m, n]}\right)_{i, i}\right|$. 


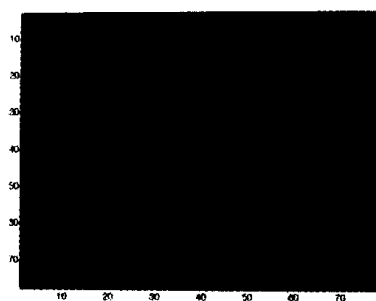

a)

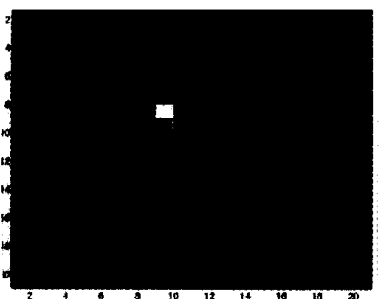

b)

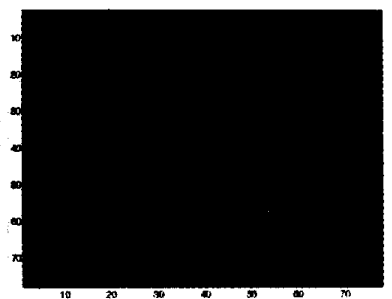

c)

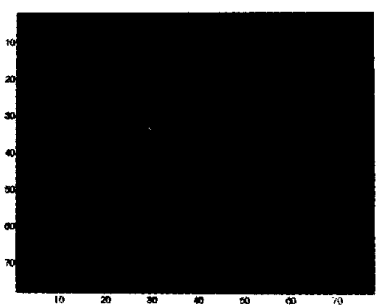

d)

Figure 4. Superresolution restoration of synthetic star example. a) Unblurred image. b) Observed blurred, noisy, subsampled image, c) MUSIC spectrum computed from b. d) Peaks from image c.

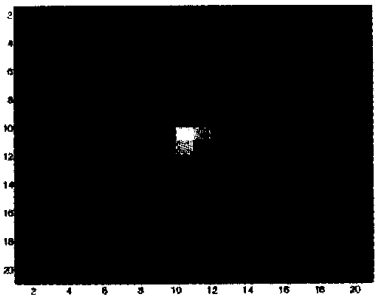

a)

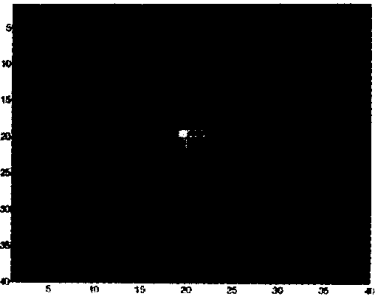

b)

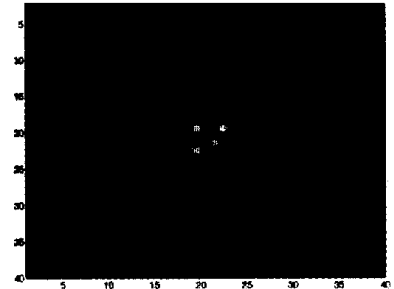

c)

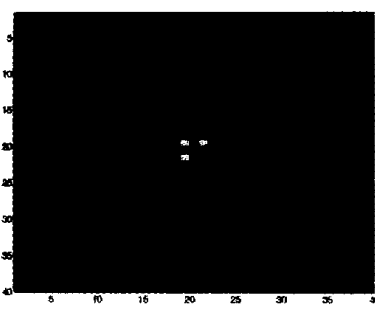

d)

Figure 5. Comparison with other algorithms. a) Blurred three star image. b) Least squares restoration, c) CLEAN restoration. d) Peaks of the MUSIC spectrum using new algorithm (same as true image.)

Transforming images into the frequency domain maps spatially shifted blurred points to a linear combination of complex sinusoids modulated by the frequency domain blurring function. This structure enables applying eigenvector based techniques, which are desirable because they allow for superresolution, approximate the ML estimator, and are computationally tractable.

Since the resulting frequency domain problem is coherent, a means of rank enhancement of the covariance function was introduced. This technique is a generalization of array smoothing that compensates for the effects of modulation introduced by the blurring function, and provides regularization to reduce noise amplification in the system. Previously, rank enhancement through smoothing had only been treated for 1-D uniform linear arrays. In the image case, smoothing may be performed despite the non-uniform modulation affects of the blur.

An expression predicting the MSE in point position estimates was derived and shown to yield a close match with large sample estimates of the error. Equation (18) is useful for evaluating performance as a function of important algorithm parameters, including the blurring function, the choice of $Q$, and the number of sub-images used in smoothing. This performance analysis and the examples of restored images demonstrate the effectiveness of the new algorithm and suggest it will be practical in a number of applications.

\section{References}

[1] M. Gunsay and B.D. Jeffs, “A subspace Decomposition Method for Point Source Localization in Blurred Image," Proc. IEEE Int. Conf. Acoust.,. Speech, Signal Processing, Apr. 1994, pp. V469V472.

[2] T. Shan, M. Wax, and T. Kailath, "On Spatial Smoothing for Direction-of-Arrival Estimation of Coherent Signals", IEEE Transactions on Acoustics, Speech and Signal Processing, Vol. 33, No. 4, pp. 806-11, Aug. 1985.

[3] M.A. Gunsay, Eigenvector Based Point Source Localization Applied to Image Restoration, Ph.D. Dissertation, Brigham Young University, July, 1994.

[4] R. 0. Schmidt, "Multiple emitter location and signal parameter estimation," in Proc. RADC Spectral Est. Workshop, Oct. 1979, pp. 243-258.

[5] B.D. Rao and K.V. S. Hari, "Effect of spatial smoothing on the performance of MUSIC and the minimumnorm method," Proc. Inst. Elec. Eng., F, Vol. 137, No. 6, pp. 449-458, Dec. 1990.

[6] J.A. Hogbom, "Aperture synthesis with a non-regular distribution of interferometer baselines," Astron. Astrophys. Suppl., vol. 15, p. 417, 1974.

[7] M. Wax and T. Kailath, "Detection of Signals by Information Theoretic Criteria," IEEE Transactions on Acoustics, Speech and Signal Processing, Vol. 33, No. 2, pp. 387-92, Apr. 1985. 